\title{
UV Mutation Abnormality
}

National Cancer Institute

\section{Source}

National Cancer Institute. UV Mutation Abnormality. NCI Thesaurus. Code C45593.

A UV Mutation Abnormality is an altered heritable state of the genetic DNA nucleotide sequence caused by exposure to ultraviolet light (UV) that results mostly in the formation of cyclobutane pyrimidine dimers and (6-4) photoproducts. UV-damaged DNA interferes with DNA replication and transcription, and can lead to cell killing or mutagenesis if the damage is not properly repaired. 